\title{
Research on Optimization of Generator Grid-Related Parameters Based on Double-Stranded Quantum Genetic Algorithm
}

\author{
Yan $\mathrm{Xu}^{1}$, Lixin Fan ${ }^{1}$, Yunqian $\mathrm{Li}^{2}$ and Zhong Chen ${ }^{2}$ \\ ${ }^{1}$ Jiangsu Fangtian Electric Technology CO. LTD, Nanjing, 211106, China \\ ${ }^{2}$ Southeast University, Nanjing, 210096, China
}

\begin{abstract}
With the expansion of grid scale and the development of UHV technology, grid partitioning mode is more and more widely applied. While the grid is closely connected with the large power grid, there is still the risk of separation and isolation mode operation. Under this background, this paper carries on the optimization research to the generation-network coordination parameters of generator set in the partitioned power network, analyzes the grid-related parameters of generator set and selects the excitation system regulator gain and the steam turbine governing system speed deviation amplification gain as the parameters optimization object. In addition, this paper takes transient stability optimization as the optimization target, deduces the objective function which shows the transient stability and establishes the corresponding optimization model. The model is solved by double-stranded quantum genetic algorithm. Finally, a practical example of the Z-S district power system of the $J$ regional grid verifies the feasibility and validity of the proposed model.
\end{abstract}

Keywords-generator grid-related parameters; grid partitioning; island operation; transient stability; double-stranded quantum genetic algorithm; machine-network coordination

\section{INTRODUCTION}

The continuous progress of various new technologies of power grid has brought the rapid development of power system. Considering the factors such as clean energy transmission and optimizing the structure of power generation resources, China's power grid system has already built an extra-high voltage transmission channel, hoping to speed up the management of air pollution, and achieving energy-saving emission reduction targets ${ }^{[1]}$.With the increasing scale of the power grid, the system operation mode is more and more complex and the network will gradually open the different voltage levels between the electromagnetic ring network to ensure stability. In order to reduce the complexity of scheduling and control power sent down to network from generator sets, special high-voltage receiving ends of the grid management using hierarchical zoning ${ }^{[2]}$. According to the United States and Canada 8.14 blackout accident report analysis, an important reason for the accident which can cause the relatively independent of the grid is that the relatively independent power grid is not established from the plantnetwork coordinated relay protection and the security and stability control system, making many generators exit from network while the system voltage drops quickly, exacerbated the occurrence of voltage collapse ${ }^{[3-4]}$. In China's smart grid planning, there is also emphasis to the necessity of plantnetwork coordinated, balanced development ${ }^{[5]}$. Therefore, in the operation and management mode of grid hierarchical partitioning, especially when there is the possibility of isolated network operation in some district grids, the rationality of turbine grid-related parameters will play a vital role in ensuring the safe and stable operation of the isolated power grid.

At present, a wide range of research on the regulation of various parameters of generator is studied at home and abroad, but there is little research on the optimization of the generator set parameters for machine-network coordination. Proportional-integral-derivative controller(referred to as PID controller) is one of the most researched aspects. The PI controller of cascaded converter with two additional loops is designed by using the harmonic search algorithm in [6],Wherein the frequency control loop causes the frequency variation to be minimized during the conversion of the grid partition from the large grid mode to the island mode operation, and the resynchronization loop can cause the power grid to be successfully reconnected; In [7], the parameters of PID controller of synchronous generator excitation control system are optimized by real-valued code genetic algorithm.; In [8], the model of generator excitation system is established and the ITSE index of terminal voltage step response curve is determined as the optimization index, finally, the PID parameters of the excitation system are optimized by MATLAB genetic algorithm toolbox. What's more, there are references pointed out that the coordination between unit protection and restriction and grid will be one of the key factors to ensure the safe and stable operation of the system ${ }^{[9-}$ ${ }^{10]}$. In addition, there are also generator parameter optimization problems in distributed generation systems, which improve the associated controller and evaluate performance ${ }^{[11]}$

Machine-network coordination refers to the power plant safety automatic devices, unit protection values, speed control system, a frequency modulation, excitation system, PSS control parameters involved in network security equipment and parameters can adapt to grid operation mode changes, so that the entire power grid of the security and stability are guaranteed $^{[12]}$. In [13], the index system of generator gridrelated parameters is established, which is divided into four main categories: primary frequency modulation, AGC, AVC, and excitation system; in [14], the uncertainties of steam turbine model parameters and the influence of various 
parameters on grid stability under different frequency disturbances are introduced; in [15], a developed generation rescheduling(GR) model is proposed, which makes the generator model less sensitive to the load pattern in the space spanned by the generator parameters and achieves the stability of the machine network.

In this paper, firstly, the selection of turbine grid-related parameters is discussed and two parameters are selected in the background of transient stability study, which are the gain of the excitation system regulator and the speed deviation amplification of the governor system. Then, based on the relevant theory, the optimization model of the adjustable turbine parameters with constraints of excitation voltage and frequency is put forward, which is based on the objective of transient stability optimization and double-chain quantum genetic algorithm. The feasibility of the optimization strategy is verified by simulation in Z-S district power system of the $\mathrm{J}$ regional grid.

\section{SELECTION OF GENERATOR GRID-RELATED PARAMETERS}

There are many parameters of the generator set, in which the parameters related to the network are a part, but there are still a considerable number. For the generator set, part of the network parameters have been preset to a specific value to achieve balance and other purposes and cannot easily adjust in the process of generating, so parameters which can be adjusted by manual adjustment or machine during operation should be selected in the selection process. In [13], the index system of generator grid-related parameters is divided into four categories: primary frequency modulation, AGC, AVC and excitation system. In this paper, the traditional governor system and excitation system adjustable parameters will be considered.

At present, in power system stability analysis, the governor model and the excitation model mainly use the standard model provided by IEEE, that is, the model used in BPA of the main power system simulation software, and then are referred to as BPA model. Here consider the use of a wide range of potential source rectifier excitation system whose BPA model is shown in Figure I; the governor system mainly considers the steam turbine governing system and the BPA model is shown in Figure II.

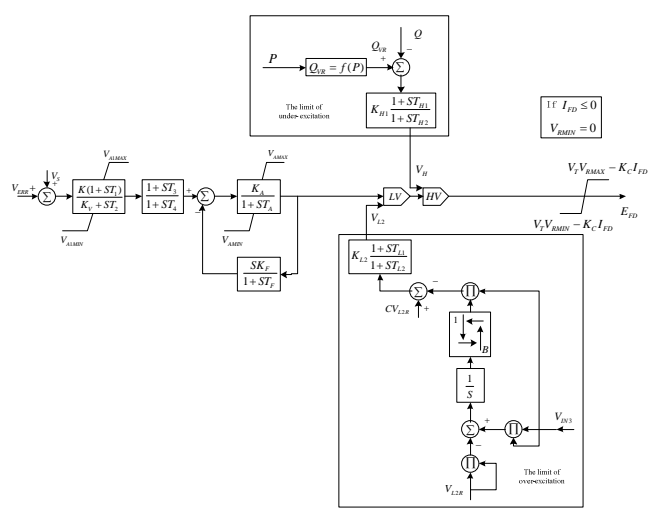

FIGURE I. THE BPA TYPE OF FV POTENTIAL SOURCE RECTIFIER EXCITATION SYSTEM

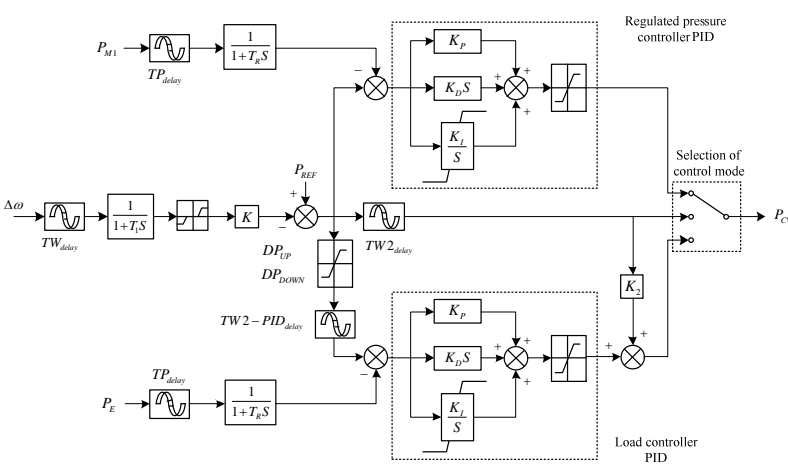

FIGURE II. THE BPA MODEL OF GJ STEAM TURBINE GOVERNING SYSTEM

Through the observation of the above two block diagram, respectively select parameters of the two models to adjust, because the two parameters do not affect the internal balance of the corresponding system, which can be adjusted to influence transient stability of the generator-connected grid when genset is in the operational state. The parameter $K$ in Figure I represents the gain of the excitation system regulator and $K V$ will be used in the following. The parameter $K$ in Figure II represents the speed deviation amplification of the governor control system, and $K J$ will be used in the following. At this point, the selection of genset optimizated parameters is completed.

\section{Optimization Model OF GenERATOR GRID-RELATED PARAMETERS}

In this paper, the transient process in the process of district grid going wrong and separated from the large power system and then operating in isolated state is taken as the main research background to observe the transient stability of the district grid, therefore, the transient stability will be the optimization target.

\section{A. Objective Function}

The stability of microgrids is discussed in detail in [17], which is summarized from four aspects: distributed microsource type, micro-source interface type, load characteristic and fault types. But it does not form a specific objective function for judging the transient stability. In this paper, the objective function of transient stability will be studied.

Under normal circumstances, when the fault occurs, the power grid will enter the transient process. This paper considers the transient process of district grid separated from the large power system. When the partitioned grid disconnects from large power system, the problem of power imbalance will be faced in the moment, and it is necessary to carry out the power rebalance by the subsequent generator set adjustment action. If the power gap of district grid is within the range of the generating capacity of generator $\operatorname{set}\left(\Delta W<\operatorname{mrg}\left(W_{g e n}\right)\right)$ and the generator set can make the correct and rapid adjustment action, the isolated grid will be able to maintain transient stability. Conversely, if the power gap of district grid exceeds the range of capacity of the 
generator $\operatorname{set}\left(\Delta W>\operatorname{mrg}\left(W_{\text {gen }}\right)\right)$, then the isolated network will lose transient stability. Transient stability judging factors are selected for two transient cases respectively.

\section{1) Transient stability}

If $W_{\text {gent }}<W_{\text {loadt }}$, that is, before the division of the district grid from large power system, the output of the generating unit $W_{\text {gent }}$ is less than all the load power $W_{\text {loadt }}$ and large power system transmits power to district grid through tie-line. After separation, the island-operated district grid will instantly get into low-frequency state and sub-regional generating units need to be additional regulated. Selecting the lowest point of frequency $f_{\min }$, the lowest point of voltage $V_{\text {min }}$ and the time of transient fluctuation in transient process $t_{s t}$ as the judgment factors, the optimal objective function for transient stability is given as follows:

$$
F_{1}=\max \left(f_{\min }+V_{\min }-t_{s t}\right)
$$

The meaning of (1) is that in transient process it requires the lowest frequency $f_{\min }$ and the lowest voltage maximum $V_{\min }$ to be maximum, (in which $f_{\min }$ and $V_{\text {min }}$ are valued of per unit and the same thereinafter), that is, the frequency and voltage declines are minimized and the time to reach the steady state $t_{s t}$ should be shortest, that is, the time for the occurrence of transient fluctuation is the shortest.

If $W_{\text {gent }}>W_{\text {loadt }}$, the district grid transmits power to large power system through tie-line on the contrary. After separation, the island-operated district grid will instantly get into highfrequency state and the output power of sub-regional generating units need to be reduced. Selecting the highest point of frequency $f_{\max }$, the highest point of voltage $V_{\max }$ and the time of transient fluctuation in transient process $t_{s t}$ as the judgment factors, the optimal objective function for transient stability is given as follows:

$$
F_{1}=\min \left(f_{\max }+V_{\max }+t_{s t}\right)
$$

\section{2) Transient instability state}

According to the above definition of transient instability state, in the event of transient instability there will be lowfrequency or high-frequency problems caused by the reason that the generator output adjustment cannot compensate the vacancy to re-stabilize the system, and finally the generator sets will be out of step and make the whole area of the system into transient instability state. Select the generator in district grid with the maximum output referenced by another generator in the same district grid to calculate the power angle difference $\Delta \delta$ as the object of observation. The moment $\Delta \delta>180^{\circ}$ is taken as the value of the instability time $t_{\text {unst }}$ and the optimal objective function for transient stability is given as follows:

$$
F_{2}=\max \left(t_{\text {unst }}\right)
$$

The meaning of (3) is that it requires the time for stability state getting into instability state $t_{\text {unst }}$ as much as possible for grid system security measures action and manual intervention to gain enough time.

\section{3) Determination of the objective function}

In an actual grid system, the ability to maintain transient stability after a fault is not invariable and the transient stability may change in different operating scenarios. When the large power system with the partitioned grid is running at the peak load scenario, the district grid which is going to disconnect with the large power system is likely to lose its stability, while the isolated network will maintain the transient stability when the grid is running at valley load scenario. The transient stability and transient instability of the two cases are combined into account to get the overall objective function as follows:

$$
F=a F_{1}+b F_{2}
$$

In (4), $a+b=1$. $a$ and $b$ are correspondingly stable factor and instable factor.

Considering the different performance of the grid under different load scenarios, we can get the value of $a$ and $b$ and then select the typical examples of transient stability and transient instability corresponding to the calculation of function $F_{1}$ and $F_{2}$. Finally, the general objective function $F$ of transient stability optimization can be calculated.

In general, if the isolated network can guarantee transient stability in various operating scenarios of the grid, then $a$ values 1 and $b$ values 0 . If the transient instability occurs in all kinds of operating scenarios of the grid, then $a$ values 0 and $b$ values 1 . For those conservative cases, $b$ can be taken as a larger value, and compared to considering the transient stability scenario it will tend to consider the transient instability optimization.

\section{B. Restrictions}

1) Excitation regulation gain constraint

$$
K V_{\min } \leq K V \leq K V_{\max }
$$

In (5), $K V$ is the regulator gain of excitation system, $K V_{\text {min }}$ and $K V_{\max }$ are correspondingly the upper and lower limits of the excitation regulation gain. The force excitation gain of the excitation system is about twice of the normal excitation magnification, so the limits can be valued by formula $K V_{\max }=2 K V_{\text {norm }}, K V_{\text {min }}=0.5 K V_{\text {norm }}$. 
2) Governor speed deviation magnification constraint

$$
K J_{\min } \leq K J \leq K J_{\max }
$$

In (6), $K J$ is speed deviation magnification times of governor control system, $K J_{\min }$ and $K J_{\max }$ are correspondingly the upper and lower limits of governor speed deviation magnification and the limits can be valued by formula $K J_{\text {max }}=2 K J_{\text {norm }}, K J_{\text {min }}=0.5 K J_{\text {norm }}$.

3) Generator set power constraint

$$
\left\{\begin{array}{l}
P_{\min } \leq P_{g e n} \leq P_{\max } \\
Q_{\min } \leq Q_{g e n} \leq Q_{\max }
\end{array}\right.
$$

In (7), $P_{\text {gen }}$ and $Q_{\text {gen }}$ are relatively active and reactive power of the generator set. $P_{\min }$ and $P_{\max }$ are upper and lower limits of active power output of generator. $Q_{\min }$ and $Q_{\max }$ are upper and lower limit of reactive power output of generator.

\section{DOUBle - STRANDED QuANTUM Genetic Algorithm}

In 1994, Shor proposed the first quantum algorithm to solve the large number factor decomposition ${ }^{[18]}$. Then the quantum algorithm has attracted much attention due to its unique computational performance; Subsequently, Narayanan et al. proposed a quantum derivative genetic algorithm ${ }^{[19]}$, which inspired the combination of quantum algorithm and genetic algorithm; and LI Shiyong et al. considered two probability chains in the quantum chromosome as the gene chain describing the optimal solution, and further proposed the double-chain quantum genetic algorithm(DCQGA) ${ }^{[20]}$, which improved the optimization performance of the algorithm further.

In this paper, the model of the application object is the district grid in large power system. In general, there are not quantities of generator sets, and the two change parameters are only taken from excitation system and speed control system. However, the calculation of transient process is complex and time-consuming is long, so the optimization calculation is still very large. In this case we need adopt the algorithm with faster searching speed. In this paper, the DCQGA algorithm is used to solve the proposed optimization model: using quantum bits to encode the chromosomes and decoding the quantum bits to the solution space by the quantum bit probability amplitude; using the quantum rotation gate to update quantum bits stepby-step, and this algorithm sees quantum probability amplitude as the two genes tied up and down so that the two chromosomes gene chains can optimize at the same time so the optimization will be speeded up; simulating the chromosomal variation process by the variation of qubit nongate to increase the population diversity and reduce the probability of premature convergence.

\section{A. Fundamentals of DCQGA}

\section{1) Quantum bit probability amplitude coding}

In quantum computing, the smallest unit of information is expressed by the quantum bit, which is known as qubit ${ }^{[21]}$. The state of a qubit can be expressed as follows:

$$
|\varphi\rangle=\alpha|0\rangle+\beta|1\rangle
$$

$\alpha$ and $\beta$ satisfies the normalization condition:

$$
|\alpha|^{2}+|\beta|^{2}=1
$$

Then a pair of complex numbers $\alpha$ and $\beta$ satisfying (8) and (9) is called the probability amplitude of a qubit. Quantum bits can also be expressed directly as $[\alpha, \beta]^{T}$.

In the DCQGA algorithm, the probability amplitude of quantum bits is directly used as the encoding. Due to the constraint in Eq.(9), the encoding is as follows:

$$
p_{i}=\left|\begin{array}{l}
\cos \left(t_{i 1}\right) \\
\sin \left(t_{i 1}\right)
\end{array}\right| \begin{gathered}
\cos \left(t_{i 2}\right) \\
\sin \left(t_{i 2}\right)
\end{gathered}|\ldots| \begin{aligned}
& \cos \left(t_{i n}\right) \\
& \sin \left(t_{i n}\right)
\end{aligned} \mid
$$

In (10), $t_{i j}=2 \pi \times r n d$, rnd is a random number between $(0,1), i=1,2, \cdots, m, j=1,2, \cdots, n \cdot m$ is the population size; $n$ is the number of qubit, and in this paper it represents the number of control variables, including the excitation and governor parameters of each generating unit. Each chromosome contains two parallel chains of genes, each of which represents a set of optimal parameters.

The two gene strands of each chromosome contains $2 n$ randomly generated variables, which are likely not to be within the constraints of parameters optimization. So try to convert the probability amplitude to the solution space by linear transformation. The $j$ th qubit on $i$ th chromosome is expressed as $\left[\alpha_{j}^{i}, \beta_{j}^{i}\right]^{T}$. Assume that $x_{i}$ is the minimum value of the corresponding parameter constraint and $y_{i}$ is the maximum value of that. The conversion equation is as follows:

$$
\left\{\begin{array}{l}
K_{j \alpha}^{i}=\frac{1}{2}\left[y_{i}\left(1+\alpha_{j}^{i}\right)+x_{i}\left(1-\alpha_{j}^{i}\right)\right] \\
K_{j \beta}^{i}=\frac{1}{2}\left[y_{i}\left(1+\beta_{j}^{i}\right)+x_{i}\left(1-\beta_{j}^{i}\right)\right]
\end{array}\right.
$$

By this linear transformation of the system in (11), the probability amplitude $\alpha_{j}^{i}$ of the quantum state $|0\rangle$ will be transformed into the solution $K_{j \alpha}^{i}$ within the parameter 
constraint and in the same way, probability amplitude $\beta_{j}^{i}$ of the quantum state $|1\rangle$ will be converted into the solution $K_{j \beta}^{i}$.

\section{2) The quantum rotation gate updating phase}

The formula for updating the qubit is as follows ${ }^{[22]}$ :

$$
\left[\begin{array}{l}
\alpha_{j}^{i+1} \\
\beta_{j}^{i+1}
\end{array}\right]=\mathbf{R}\left[\begin{array}{c}
\alpha_{j}^{i} \\
\beta_{j}^{i}
\end{array}\right]
$$

In (12), $\left[\alpha_{j}^{i}, \beta_{j}^{i}\right]^{T}$ and $\left[\alpha_{j}^{i+1}, \beta_{j}^{i+1}\right]^{T}$ are correspondingly the $j$ th qubit of chromosome generation $i$ and generation $i+1$. The quantum rotation gate $\mathbf{R}$ is expressed as follows:

$$
\mathbf{R}=\left[\begin{array}{rr}
\cos \theta & -\sin \theta \\
\sin \theta & \cos \theta
\end{array}\right]
$$

In [21], an improved scheme of angle step function is proposed, which will be defined in the same way in this paper:

$$
\Delta \theta_{i j}=-\operatorname{sgn}(A) \times \Delta \theta_{0} \times \exp \left(-\frac{\left|\nabla f\left(X_{j}^{i}\right)\right|-\nabla f_{j \min }}{\nabla f_{j \max }-\nabla f_{j \min }}\right)
$$

In (14), sgn is the symbolic function. $A$ is defined as follows:

$$
A=\left|\begin{array}{cc}
\cos \theta_{0} & \cos \theta_{1} \\
\sin \theta_{0} & \sin \theta_{1}
\end{array}\right|=\sin \left(\theta_{1}-\theta_{0}\right)
$$

According to the definition of the qubit phase in [23], $\theta \in[-\pi / 2, \pi / 2], \Delta \theta_{0}$ is valued as $0.5 \pi . \theta_{0}$ and $\theta_{1}$ respectively represent the qubit probability amplitudes of global optimal solution and current solution. $\nabla f\left(X_{j}^{i}\right)$ is the gradient of the objective function $f(x)$ at point $X_{j}^{i}$. Since that most problems of power system is discrete and the researched issue is also discrete, and the numerator and denominator of the formula are both forms of gradient, this paper uses the first difference between two generations to replace the original formula. The redefined formula is as follows:

$$
\nabla f\left(X_{j}^{i}\right)=f\left(X_{j}^{i+1}\right)-f\left(X_{j}^{i}\right)
$$

$$
\begin{aligned}
\nabla f_{j \max }= & \max \left\{\left|f\left(X_{j}^{2}\right)-f\left(X_{j}^{1}\right)\right|,\right. \\
& \left.\cdots,\left|f\left(X_{j}^{m}\right)-f\left(X_{j}^{m-1}\right)\right|\right\}
\end{aligned}
$$

$$
\begin{aligned}
\nabla f_{j \min }= & \min \left\{\left|f\left(X_{j}^{2}\right)-f\left(X_{j}^{1}\right)\right|,\right. \\
& \left.\cdots,\left|f\left(X_{j}^{m}\right)-f\left(X_{j}^{m-1}\right)\right|\right\}
\end{aligned}
$$

In (16)-(18), $X_{j}^{i}$ represents the $j$ th qubit in chromosome $i$. The $i+1$ generation chromosome is the parent chromosome of the progeny chromosome $i$.

The size and direction of the angle $\Delta \theta$ can be determined by the angle step function. Using this method, the change trend of the objective function at the search point can be added to the calculation of angle step. When the rate of change is large, the angle step will be reduced while the rate of change is small, the angle step can be increased moderately. With the intelligent and flexible step change, the search process will be speeded up while not miss the optimal solution at the same time.

\section{3) Quantum bit non-gate variation}

The objective function value of each chromosome is defined as fitness in iteration process, and the average fitness of the new-scale population after each iteration is $f_{\text {avg }}=\sum_{i=1}^{m} f_{i} / m$. The average of the fitness value greater than $f_{\text {avg }}$ is computed as $f_{\text {avg }}{ }^{\prime}$, and define that $\Delta f=\left|f_{\text {avg }}-f_{\text {avg }}{ }^{\prime}\right|$. If $\Delta f$ is marginal, it indicates that the population tends to be premature and the diversity is destroyed. Therefore, in the genetic iterative optimization process, considering increasing the diversity of the population is necessary, that is, the introduction of the concept of qubit non-gate variation.

According to the improvement of this step in [21], when the algorithm is operating, a certain mutation probability is applied to each generation of chromosomes. When the mutation occurs, randomly select several qubits to apply the quantum non-gate transform, and the possibility amplitudes of the two qubits involved in the transformation are interchanged, so that the two gene chains can be mutated simultaneously. This mutation is actually a positive rotation of the quantum bit amplitude angle, which simulates the phenomenon of genetic variation and can effectively reduce the risk of population precocity.

\section{B. Parameter Optimization Process Based on DCQGA}

To sum up, with the objective function of transient stability optimization, DCQGA-based optimization process of generator grid-related parameters is summarized as follows: 
a) Obtain the detailed information of lines, load nodes and generating units and input the original data of the system;

b) Initialize the population and set iteration times as zero;

c) Apply the mutation probability to the current chromosome, and conduct the qubit non-gate variation if the mutation occurs;

d) Decode the individual data of the current chromosome into solution space and transfer it from the binary to the real number to determine whether each parameter satisfies the constraint conditions of the function; if so, the parameters will be substituted into the transient stability calculation program and the correlation calculation is carried out;

e) Evaluate the fitness of the optimal objective function value of the transient stability calculated in the last step and record the current optimal value;

f) Calculate the quantum rotation angle and update the quantum gate to obtain the next generation chromosome;

g) If the number of iterations has reached the preset population size, the optimal result recorded in the iteration will be output and the iterative process will be finished. Otherwise, the number of iterations is added by one and the process returns to the step (c) to continue the iteration.

\section{COMPUTATIONAL ANALYSIS OF EXAMPLES}

The actual operation data from Z-S district power system in $\mathrm{J}$ regional grid is used as an example to verify the feasibility and correctness of the above model. In the study, the operating modes of this district are divided into six types, which are winter valley-load scene, winter peak-load scene, winter shoulder-load scene, summer valley-load scene, summer peakload scene and summer shoulder-load scene. After simulating by software BPA, it indicates that there are three types of operation modes to maintain the transient stability while the other three types getting into transient instability in the process of district grid being separated from large power system due to the fault, so set $a=b=0.5$ in the objective function $F$. In addition, in the simulation of power flow calculation, it is found that in all operation modes, when the regional power grid is connected with the large power grid, the power is transferred from the large power grid along the transmission line to the zonal power grid. Therefore, when the tie line fails, use the low-frequency objective function.

In the example grid, there are four $220 \mathrm{kV}$ power plants and correspondingly named No.1 to No.4. Solve the model by DCQGA. The convergence process of the objective function is shown in Figure III as the algebra increases in the iterative process.

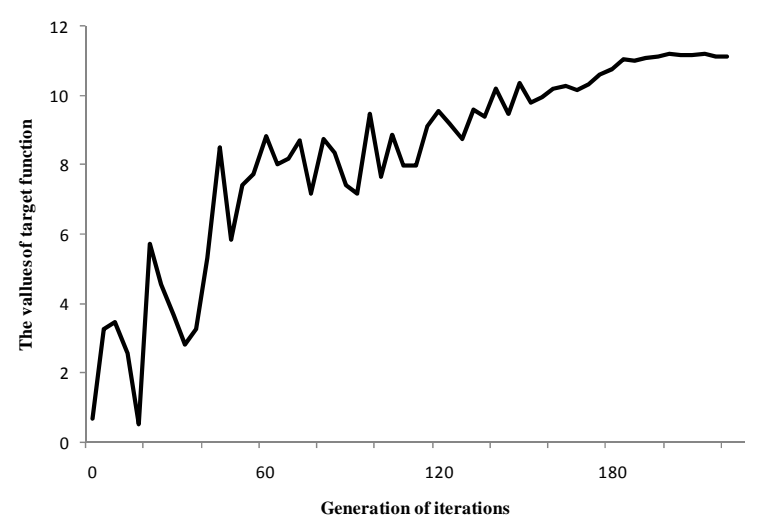

FIGURE III. THE CONVERGENCE PROCESS OF DCQGA

As the population size grows and the number of iterative algebras increases, the objective function of the system tends to show a fluctuant rising process. As the gene doublestranded search simultaneously, the convergence rate is very fast and the result will become steady from the 190th generation. The comparison of original and optimal data of generator grid-related parameters is shown in Table I.

TABLE I. THE ORIGINAL AND OPTIMAL DATA OF GENERATOR GRID-RELATED PARAMETERS

\begin{tabular}{ccccccccc}
\hline & $\begin{array}{c}\text { Station } \\
\text { 1's KV }\end{array}$ & $\begin{array}{c}\text { Station } \\
\text { 1's KJ }\end{array}$ & $\begin{array}{c}\text { Station } \\
\text { 2's KV }\end{array}$ & $\begin{array}{c}\text { Station } \\
\text { 2's KJ }\end{array}$ & $\begin{array}{c}\text { Station } \\
\text { 3's KV }\end{array}$ & $\begin{array}{c}\text { Station } \\
\text { 3's KJ }\end{array}$ & $\begin{array}{c}\text { Station } \\
\text { 4's KV }\end{array}$ & $\begin{array}{c}\text { Station } \\
\text { 4's KJ }\end{array}$ \\
\hline Origin & 2.75 & 11.7 & 500 & 11.7 & 500 & 11.7 & 500 & 11.7 \\
Optimization & 2.94 & 21.1 & 431.27 & 19.8 & 472.51 & 19.52 & 744.7 & 22.4 \\
\hline
\end{tabular}

According to the original data and the optimal solution, construct and analyze the difference between transient stability frequency fluctuations of the district grid operating in isolated mode in the two situations and it is shown in Figure IV.

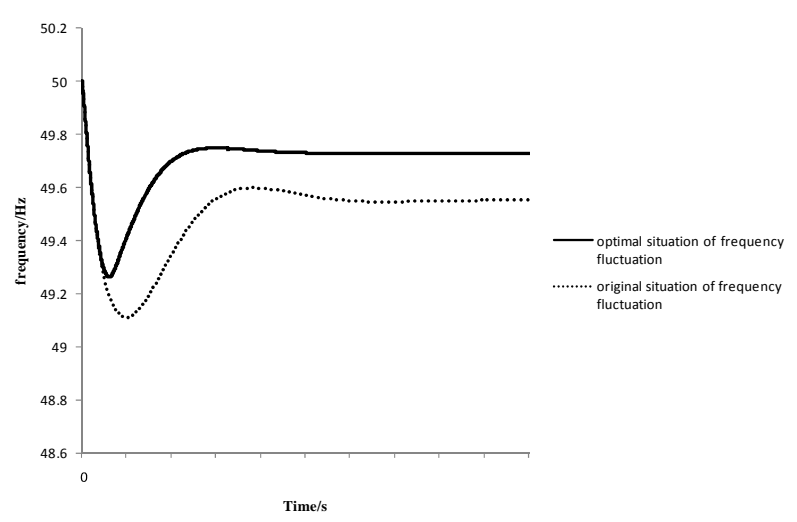

FIGURE IV. THE COMPARISON OF TRANSIENT FLUCTUATION DURATIONS IN SITUATIONS OF OPTIMAL AND ORIGINAL PARAMETERS

In Figure IV, it is easy to find that the optimal combination of parameters has a shorter transient fluctuation time and a smaller fluctuation, which is better than the combination of the original parameters in transient stability. It shows that the optimal solution obtained by solving the model plays an important role in optimizing grid-related parameters. Through 
the simulation of BPA software and DCQGA algorithm solving the model, the optimal combination of the grid-related parameters of power plant can be gained and the feasibility and correctness of the proposed model will be proved.

\section{CONCLUSION}

Scale expansion of power grid and the construction of the UHV power grid will bring development and convenience, but also bring many challenges, such as grid hierarchical partition operation and management. In the case of hierarchical partition operation, the partitioned power grid may face the risk of being separated from the large power grid with a very small but non-zero probability, in which case it is very important to ensure the optimal transient stability of the partitioned power grid. In order to meet the requirements of machine-network coordination, the optimization research of turbine grid-related parameters has become an important issue.

In this paper, two parameters of excitation system and speed control system are selected as the optimization object of the turbine grid-related parameters and the expression of the objective function of transient stability is deduced. According to the above work, the model is established and the fast and effective double-stranded quantum genetic algorithm is used to solve it. A practical example of Z-S district power system in the $\mathrm{J}$ regional grid shows that the model and the algorithm proposed in this paper can reasonably and effectively optimize the grid-related parameters of the power plant and improve the transient stability of the regional power grid. However, there are still many places to be improved. For example, the choice of optimized parameters and the expression of transient stability function still exists other ways, which need further study.

\section{ACKNOWLEDGMENT}

Project supported by State Grid Corporation of China science and technology project 'Research on lay-dividing and zone-dividing mode and isolated network operation of Jiangsu power grid in condition of UHV AC/DC multi-set system'.

\section{REFERENCES}

[1] LIU Zhenya, ZHANG Qiping, DONG Cun, et al. Efficient and security transmission of wind, photovoltaic and thermal power of large-scale energy resource bases through UHVDC projects[J]. Proceedings of the CSEE, 2014, 34(16): 2513-2522.

[2] JIANG Mu, WU Wenchuan, ZHANG Boming, et a1. A multi-area coordinated power dispatch method for provincial power grid[J]. Automation of Electric Power Systems, 2009, 33(22): 1-5.

[3] YIN Yonghua, GUO Jianbo, ZHAO Jianjun, et al. Preliminary analysis of large scale blackout in interconnected North America power grid on August 14 and lessons to be drawn[J]. Power System Technology, 2003, 27(10): 8-11.

[4] GAN Deqiang, HU Jiangyi, HAN Zhenxiang. Thinking over several blackouts in 2003[J]. Automation of Electric Power Systems, 2004, 28(3): 1-4, 9(in Chinese)

[5] XIAO Shijie. Consideration of technology for constructing Chinese smart grid[J]. Automation of Electric Power Systems, 2009, 33(9): 1-4.

[6] M. N. Ambia, H. M. Hasanien, A. Al-Durra and S. M. Muyeen, "Harmony Search Algorithm-Based Controller Parameters Optimization for a Distributed-Generation System," in IEEE Transactions on Power Delivery, vol. 30, no. 1, pp. 246-255, Feb. 2015
[7] R. Ramya, K. Selvi and S. S. Nivethitha, "Optimization of synchronous generator excitation controller parameters," Power, Energy and Control (ICPEC), 2013 International Conference on, Sri Rangalatchum Dindigul, 2013, pp. 585-590.

[8] Y. Cao and J. Ma, "Research on PID parameters optimization of synchronous generator excitation control system," Critical Infrastructure (CRIS), 2010 5th International Conference on, Beijing, 2010, pp. 1-5.

[9] YUAN Liang, WU Guoyang, YANG Mingyu, et al. The grid-related relay protection and limited automatic check of genset based on full dynamic simulation[J]. Automation of Electric Power Systems, 2015, 39(24): 115-121.

[10] JI Keqin. Research on relay protection and automatic equipment parameters optimization of large-scale genset based on machine-network cordination[D]: [master's thesis]. Shanghai: Shanghai Jiaotong University, 2007.

[11] A. Timbus, M. Liserre, R. Teodorescu, P. Rodriguez and F. Blaabjerg, "Evaluation of Current Controllers for Distributed Power Generation Systems," in IEEE Transactions on Power Electronics, vol. 24, no. 3, pp. 654-664, March 2009.

[12] LI Miao. Research on a number of issues of power system machinenetwork coordination[D]: [doctoral dissertation]. Wuhan: Wuhan University, 2012.

[13] CHEN Yidong, CHENG Haozhong, LUAN Weijie, ZHANG Xiaochen. Online evaluation of generator grid-related parameters[J]. East China Power, 2013, 41(4): 789-793.

[14] XU Wei, HE Qing, HUANG Baohua, QIU Xiaozhi. Research on Frequency Domain Analysis on the Uncertainty of Parameters of Steam Turbine Model Related to Grid[J]. North China Electric Power, 2014(06): 20-23.

[15] N. Xiong et al., "Investigation on Limit Surfaces in Space Spanned by Generation Parameter," in IEEE Transactions on Power Systems, vol. 25, no. 3, pp. 1309-1318, Aug. 2010.

[16] XU Wei. Modeling and parameters adaptation research of steam turbine and speed governor system[D]: [master's thesis]. Beijing: North China Electric Power University, 2015.

[17] SHUAI Zhikang, ZOU Fuxiao, TU Chunming, et al. Review on transient stability of microgrid[J]. Automation of Electric Power System, 2015, 39(16): 151-159(in Chinese).

[18] P. W. Shor, "Algorithms for quantum computation: discrete logarithms and factoring," Foundations of Computer Science, 1994 Proceedings., 35th Annual Symposium on, Santa Fe, NM, 1994, pp. 124-134.

[19] A. Narayanan and M. Moore, "Quantum-inspired genetic algorithms," Evolutionary Computation, 1996., Proceedings of IEEE International Conference on, Nagoya, 1996, pp. 61-66.

[20] LI Shiyong, LI Panchi. Quantum evolutionary algorithm based on real coding and gradient information of object function[J]. Journal of Harbin Institute of Technology, 2006, 38(8): 1216-1219.

[21] XU Shaohua, XU Chen, HAO Xing, WANG Ying, LI Panchi. Improved quantum genetic algorithm with double chains and its application[J]. Application Research of Computers, 2010, 27(6): 2090-2092.

[22] LIU Hongwen, ZHANG Gexiang. A reactive power optimization method based on improved quantum-inspired genetic algorithm[J]. Power System Technology, 2008, 32(12): 35-38.

[23] ZHANG Gexiang, LI Na, JIN Weidong, et al. A novel quantum genetic algorithm and its application[J]. Acta Electronica Sinica, 2004, 32(3): 476-479(in Chinese). 